\title{
Evaluation of Tissue Stem Cell-Derived Human Intestinal Organoids, a Physiologically Relevant Model to Evaluate Cytochrome P450 Induction in Gut ${ }^{\mathbb{\Phi}}$
}

\author{
David M. Stresser, Jun Sun, and Sarah S. Wilson
}

AbbVie, Inc., North Chicago, Illinois (D.M.S., J.S.) and AbbVie Cambridge Research Center, Cambridge, Massachusetts (S.S.W.)

Received October 9, 2020; accepted December 1, 2020

\section{ABSTRACT}

Induction of cytochrome P450 can cause drug-drug interactions and efficacy failure. Induction risk in liver and gut is typically inferred from experiments with plated hepatocytes. Organoids are physiologically relevant, multicellular structures originating from stem cells. Intestinal stem cell-derived organoids retain traits of normal gut physiology, such as an epithelial barrier and cellular diversity. Matched human enteroid and colonoid lines, generated from ileal and colon biopsies from two donors, were cultured in extracellular matrix for 3 days, followed by a single 48-hour treatment with rifampin, omeprazole, CITCO, and phenytoin at concentrations that induce target genes in hepatocytes. After treatment, mRNA was analyzed for induction of target genes. Rifampin induced CYP3A4; estimated $\mathrm{EC}_{50}$ and maximal fold induction were $3.75 \mu \mathrm{M}$ and 8.96-fold, respectively, for ileal organoids and $1.40 \mu \mathrm{M}$ and 11.3-fold, respectively, for colon organoids. Ileal, but not colon, organoids exhibited nifedipine oxidase activity, which was induced by rifampin up to 14-fold. The test compounds did not increase mRNA expression of CYP1A2, CYP2B6, multidrug resistance transporter 1 (P-glycoprotein), breast cancer resistance protein, and UDP-glucuronosyltransferase 1A1 in ileal organoids. Whereas omeprazole induced CYP3A4 (up to 5.3-fold, geometric mean, $n=4$ experiments), constitutive androstane receptor activators phenytoin and CITCO did not. Omeprazole failed to induce CYP1A2 mRNA but did induce CYP1A1 mRNA (up to 7.7-fold and 15-fold in ileal and colon organoids, respectively, $n=4$ experiments). Despite relatively high intra- and interexperimental variability, data suggest that the model yields induction responses that are distinct from hepatocytes and holds promise to enable evaluation of CYP1A1 and CYP3A4 induction in gut.

\section{SIGNIFICANCE STATEMENT}

An adult intestinal stem cell-derived organoid model to test P450 induction in gut was evaluated. Testing several prototypical inducers for mRNA induction of P450 isoforms, UDP-glucuronosyltransferase 1A1, P-glycoprotein, and breast cancer resistance protein with both human colon and ileal organoids resulted in a range of responses, often distinct from those found in hepatocytes, indicating the potential for further development of this model as a physiologically relevant gut induction test system.

\section{Introduction}

Induction of cytochrome P450 enzymes by a drug candidate may lead to unwanted increases in the rate of metabolism and elimination of itself or comedications. Although induction may occur in multiple tissues,

Genotype-Tissue Expression (GTEx) Project was supported by the Common Fund of the Office of the Director of the National Institutes of Health and by National Cancer Institute, National Human Genome Research Institute, National Heart, Lung, and Blood Institute, National Institute on Drug Abuse, National Institute of Mental Health, and National Institute of Neurological Disorders and Stroke (https://commonfund.nih.gov/about). The data used for the analyses described in this manuscript were obtained from the GTEx Portal on November 14, 2020.

All authors are employees of AbbVie and may own AbbVie stock. AbbVie sponsored and funded the study; contributed to the design; participated in the collection, analysis, and interpretation of data; and participated in writing, reviewing, and approval of the final publication.

Portions of this work were originally presented at: Stresser DM, Sun J, Patnaude L, Terrillon S, and Wilson SS (2019) Tissue stem cell-derived human intestinal organoids: A novel and physiologically relevant model to evaluate cytochrome P450 induction in gut. 12th International ISSX Meeting; 2019 Jul 28-31; Portland, OR

https://doi.org/10.1124/dmd.120.000281.

SThis article has supplemental material available at dmd.aspetjournals.org. liver and gut are tissues for which induction is considered quantitatively meaningful in affecting pharmacokinetics. Preclinical or in vitro methods to evaluate induction in gut are generally lacking. Therefore, the prediction of gut induction response is typically inferred from studies conducted in plated human hepatocytes. This practice is typically applied to CYP3A4 inducers only, as the enzyme is expressed and inducible in both liver and gut. However, some compounds, such as efavirenz and carbamazepine, have been shown to induce CYP3A in liver but not in gut (Mouly et al., 2002; Giessmann et al., 2004; Meyer zu Schwabedissen et al., 2012), indicating that a hepatocyte model for induction may not always be appropriate. One explanation for these observations is that enterocytes respond differently to inducing agents compared with hepatocytes. Indeed, the identity and relative abundance of enzymes and transporters expressed in gut are distinct from that in liver, suggesting that gene expression and induction regulatory mechanisms may differ between the two tissues. For example, expression of CYP2C8, CYP1A2, and CYP2B6, although significant in liver, is generally reported absent in gut (Paine et al., 2006; Drozdzik et al., 2018). Conversely, expression of CYP1A1 and UGT1A10 is observed in gut but not in liver (Paine et al., 2006; Oda et al., 2017). Since the fraction of drug absorbed and metabolized in the gut wall is a critical component of overall oral bioavailability, evaluating the 
inducibility of drug-metabolizing enzymes and transporters in models derived from intestine, rather than from liver, would be desirable.

Drug oral bioavailability $(F)$ may be described as the fraction of oral drug escaping gut metabolism and transporter-mediated secretion $\left(F_{\mathrm{g}}\right)$ multiplied by the fraction absorbed $\left(F_{\mathrm{a}}\right)$ and the fraction metabolized in liver or excreted into bile. In a literature survey of $>300$ compounds with abundant human pharmacokinetic data, approximately $30 \%$ exhibited $F_{\mathrm{g}}$ of 0.8 or less (Varma et al., 2010), demonstrating the important role of gut metabolism in estimating $F$. Many compounds exhibiting a substantial $F_{\mathrm{g}}$ component are metabolically unstable and are substrates of CYP3A4 and efflux transporters (Zhang and Benet, 2001). Therefore, induction of CYP3A4 and/or efflux transporters in gut could affect $F_{\mathrm{g}}$ and mediate drug-drug interactions (Galetin et al., 2010; Alqahtani et al., 2018). Attempts to evaluate P450 induction in gut using colon carcinoma-derived cell lines have met with limited success and are generally considered of questionable relevance. The Caco2 cell line is widely deployed to study intestinal permeability and transport, but it is not suitable for evaluating CYP3A4 induction and regulation because it lacks PXR (Thummel et al., 2001; Schmiedlin-Ren et al., 2001; Hartley et al., 2006; Brück et al., 2017). Another colon carcinoma cell line, LS-180, is responsive to PXR-mediated inducers but may exhibit atypical gene expression while lacking the advantages of primary cells (Schuetz et al., 1996; Gupta et al., 2008; Zheng et al., 2012). Recently, cryopreserved human intestinal mucosal epithelium, consisting of multicellular fragments from villi of multiple donors, has become commercially available (Li et al., 2018). Incubation of these fragments with rifampin or 1,25-dihydroxyvitamin D3 resulted in up to a 3-fold induction of CYP3A4 mRNA, thereby offering a model using human tissue-derived material. Potential limitations of this model are the dynamic range of the induction response and longevity of the cultures upon thawing, which are apparently 3 -fold and up to 24 hours, respectively.

Advanced microphysiological systems and three-dimensional tissue culture models continue to gain in popularity. By incorporating attributes such as multiplicity of cell types, three-dimensional architecture, flow, and mechanical motion, in vivo-like function is purported to be superior to conventional two-dimensional cell culture models (Madden et al., 2018; Fowler et al., 2020). Organoids are tissuemimetic, microphysiological, three-dimensional structures that are experimentally derived from stem cells (Fatehullah et al., 2016). Human intestinal organoids derived from intestinal stem cells retain several traits of normal gut physiology, mirroring the microenvironment and function of the epithelial barrier as well as the epithelial cell diversity of the gastrointestinal tract. These models have recently gained interest from drug metabolism scientists evaluating induction and other absorption, distribution, metabolism, and excretion parameters. Onozato et al. (2018) used an iPS cell-derived intestinal organoid model to evaluate induction of CYP3A4 and other enzymes. In this study, after the 31-day culture and differentiation period, a single 3-day treatment of $40 \mu \mathrm{M}$ rifampin was given and induced CYP3A4 mRNA 2-fold. Authors concluded there was a need to develop an improved differentiation protocol to increase induction response levels. More recently, adult stem cell-derived duodenal organoids, isolated into fragments and seeded to form monolayers on polydimethylsiloxane chips, were used to demonstrate a 5.3 -fold induction of CYP3A4 mRNA by $20 \mu \mathrm{M}$ rifampin treatment (Kasendra et al., 2020).

Human ileal and colon organoid cultures have been established as pharmacology models in our laboratory using adult tissue-resident stem cells based on methods developed previously (VanDussen et al., 2015). In this investigation, the effect of several prototypical inducers on the mRNA expression of CYP3A4 and other P450 isoforms, UGT1A1, Pgp, and BCRP with both human colon and ileal organoids was investigated. We demonstrate that the model can exhibit significant induction responses ( $>10$-fold, depending on inducer and target) that are dependent on drug concentration. Responses for some compounds were often distinct from those found in hepatocytes and indicate the potential for further development of this model as a physiologically relevant gut induction test system.

\section{Materials and Methods}

Organoid Establishment and Culture. Established human ileal and colon organoid lines were obtained from the Stappenbeck and Ciorba laboratories at the University of Washington in St. Louis (VanDussen et al., 2015; VanDussen et al., 2019). Lines were derived from biopsy samples from donors of unknown sex; the collection and use of human intestinal tissue for spheroid culture was approved by the Institutional Review Board of Washington University School of Medicine, and written informed consent was obtained from the donors prior to inclusion in the study. For routine culture, organoids were grown in 15- $\mu$ l plugs of growth factor-reduced Matrigel (Corning, Corning, NY) in 48-well plates and overlaid with $350 \mu \mathrm{l}$ of growth media containing Wnt3a, R-spondin3, and Noggin. Growth media comprised Advanced DMEM/F12 (12634-010; ThermoFisher Scientific), $1 \times$ GlutaMAX (35050; ThermoFisher Scientific), $1 \times$ penicillin/streptomycin (15140; ThermoFisher Scientific), and 20\% FBS (F2442; Sigma), to which was added at a 1:1 ratio conditioned media derived from L-WRN cell line (CRL-3276; ATCC), $10 \mu \mathrm{M}$ Y-27632 (Rho-associated protein kinase inhibitor; Tocris, Minneapolis, MN), and $10 \mu \mathrm{M} \mathrm{SB}-431542$ (transforming growth factor- $\beta$ type I receptor inhibitor; Tocris). Organoids were passaged every 3 to 4 days using mechanical dissociation and trypsin (Millipore Sigma). The production and quality control of L-WRN conditioned media produced from the L-WRN cell line, as well as its utilization to support human ileal and colon organoid culture, have been described in detail elsewhere (Miyoshi and Stappenbeck, 2013; VanDussen et al., 2019). For this study, conditioned medium collected on each day of days $1-4$, filtered using $0.22-\mu \mathrm{m}$ polyethersulfone filters and frozen, was used in growth media for all assays.

Organoid Model and Induction Experiments. To normalize organoid seeding for assays, after trypsinization and manual pipetting for disruption into single cells, organoids were filtered through a $40-\mu \mathrm{m}$ filter and spun down in growth media. Organoid pellets were then resuspended in $1 \mathrm{ml}$ of the growth media, and a $15-\mu 1$ aliquot of this sample was removed and then diluted 2-fold up to a 1:16 dilution in growth media. In total, $10 \mu$ l of this organoid suspension was then added to a black-walled, clear-bottom, 96-well plate containing $100 \mu \mathrm{l}$ CellTiter-Glo 3D (G9683; Promega) $+100 \mu$ l of PBS. The plate was protected from light and incubated at room temperature for 30 minutes, with shaking for the first 5 minutes. During incubation, a stock solution of colonoids was kept in growth media on ice. For plating, the solution of colonoids in washing media was spun at 5 minutes, $600 \mathrm{~g}$, ambient temperature, in a 50-ml conical tube as above. Following aspiration, the pellet was resuspended in the appropriate volume of thawed Matrigel, with the dilution dependent on normalization to a preselected value based on luminescence readings. The preferred density for plating and corresponding luminescence value was experimentally evaluated and is dependent on the machine used to read luminescence. Once solidified, Matrigel

ABBREVIATIONS: AhR, aryl hydrocarbon receptor; BCRP, breast cancer resistance protein; CAR, constitutive androstane receptor; $\mathrm{Cl}$, confidence interval; CITCO, 6-(4-chlorophenyl)imidazo[2,1-b][1,3]thiazole-5-carbaldehyde O-(3,4-dichlorobenzyl)oxime; Ct value, cycle threshold value; EGF, epidermal growth factor; geomean, geometric mean; $F_{g}$, fraction of oral drug escaping gut metabolism and transporter secretion; $\mathrm{P} 450$, cytochrome P450; $E_{\max }$, maximal fold induction; iPS, induced pluripotent stem; NR1/2, nuclear receptor subfamily 1 group I member 2; NR1/3, nuclear receptor subfamily 1 group I member 3; Pgp, P-glycoprotein; PXR, pregnane X receptor; RT-PCR, real-time polymerase chain reaction; UGT, UDPglucuronosyltransferase; Wnt3a, Wnt family member 3A; WRN, Wnt3a R-spondin Noggin. 
plugs were overlaid with growth media as described above but with the addition of $100 \mathrm{nM}$ dexamethasone. After three days, organoid growth media was replaced with Advanced DMEM/F12 containing L-glutamine, penicillin/streptomycin, $50 \mathrm{ng} / \mathrm{ml}$ recombinant human EGF (Thermo Fisher Scientific), $100 \mathrm{nM}$ dexamethasone, and test inducer compounds at indicated concentrations or DMSO vehicle control.

Enzyme Activity. CYP3A4 enzyme activity was determined by measuring oxidation of nifedipine after 48 hours of treatment of organoids with rifampin or solvent vehicle control. Following media removal by aspiration, organoid plugs were washed once with PBS before being overlaid with $300 \mu$ l Advanced DMEM/F12 containing L-glutamine, penicillin/streptomycin, $50 \mathrm{ng} / \mathrm{ml}$ recombinant human EGF, and 5 or $50 \mu \mathrm{M}$ nifedipine. A time zero sample (150 $\mu \mathrm{l})$ was removed, and after incubating 60 or 360 minutes at $37^{\circ} \mathrm{C}$, the remaining $150 \mu \mathrm{l}$ was collected. Dehydronifedipine was quantified by liquid chromatography-mass spectrometry using verapamil as an internal standard.

Viability Assessment. To assess organoid survival after inducer treatment, media was aspirated, and Matrigel plugs were overlaid with $100 \mu \mathrm{l}$ of CellTiter GLO 3D and $100 \mu \mathrm{l}$ of PBS, incubated while shaking for 30 minutes at ambient temperature, and then volume-transferred to a 96-well clear-bottom black well plate for luminescence reading on an Envision 2105 Multimode plate reader.

RNA Isolation. After inducer treatments (and nifedipine incubations, if applicable), Matrigel plugs were washed with PBS, resuspended in RNAlater (Thermo Fisher Scientific), and kept frozen $\left(-80^{\circ} \mathrm{C}\right)$ for subsequent RNA isolation. The mRNA from organoids was extracted with MagMax-96 Total RNA isolation kit.

RT-PCR. In previous investigations (and shown in Fig. 8 for $18 \mathrm{~S}$ values), we observed consistent recovery of mRNA across well plates. Therefore, after RNA extraction, a fixed volume of total RNA was reverse-transcribed into cDNA (SuperScript VILO cDNA-synthesis kit). RT-PCR was performed on ViiA7 or QuantStudio real-time polymerase chain reaction system using TaqMan Assays-on-Demand Gene Expression assays to assess expression of target genes and normalized to the housekeeping gene GAPDH or, for NR1I2 (PXR) and NR1I3 (CAR) only, 18S ribosomal RNA. The following TaqMan Gene expression assay primer/probe sets were used: CYP3A4, Hs00604506_m1; CYP1A1, Hs02382618_s1; CYP1A2, Hs00167927_m1; CYP2B6, Hs03044634_m1; UGT1A1, Hs02511055_s1; P-gp, Hs01394345_g1; BCRP, Hs01053790_m1; GAPDH, Hs02786624_g1; NR1I2, Hs_00901571; NR1I3, Hs00901571_m1, 18S ribosomal RNA, Hs99999901_s1.

RNA-Sequence Expression Analysis. Tissue expression data are from the Genotype-Tissue Expression Portal (https://gtexportal.org/home/; GTEx Consortium 2013), accessed on November 14, 2020, and expressed as transcripts per million.

Statistical Analysis. For nonparametric data, statistical significance between groups was conducted using Kruskal-Wallis nonparametric test within Prism software, followed by Dunn's multiple comparisons if statistical significance ( $\alpha=$ 0.05 ) was indicated. Unpaired $t$ tests were used to compare the effect of rifampin on nifedipine oxidase activities in ileal organoids.

Curve Fitting. The $\mathrm{EC}_{50}$ and $\mathrm{E}_{\max }$ values were generated by nonlinear curve fitting using a four-parameter model: $\mathrm{Y}=\mathrm{Bottom}+\left(\mathrm{X}^{\wedge} \text { Hillslope }\right)^{*}($ Top-Bottom $) /$ $\left(\mathrm{X}^{\wedge}\right.$ HillSlope $+\mathrm{EC}^{\wedge} 0^{\wedge}$ HillSlope $)$ with the bottom constrained to 1 .

\section{Results}

Establishing the Model. When intestinal organoids are grown in Wnt3a-containing media, such as the Wnt3a-R-spondin-Noggin (WRN) conditioned media used in this study, they are stem cell-enriched and characterized by their cystic appearance with thin cell layers and large lumens (VanDussen et al., 2015). Upon removal of Wnt from the culture media, stem cells rapidly differentiate into epithelial cell types, including goblet cells, enteroendocrine cells, and enterocytes (Sato et al., 2011). To establish a model to look at induction of drug-metabolizing enzymes in human enterocytes, organoids were grown for 3 days in WRN conditioned media + dexamethasone to allow for stem cell expansion. This was followed by 2 days of growth in media (Advanced DMEM/F12 containing L-glutamine, penicillin/streptomycin/dexamethasone) containing EGF as the only growth factor (media + EGF in figures) in the presence or absence of the inducers. It should be noted that the choice to include dexamethasone in the media was based on previous findings that a glucocorticoid agonist is required for robust manifestation of CYP3A4 induction in hepatocytes (Pascussi et al., 2000). Whether it is required for CYP3A4 induction in this test system is not yet known. Medium supplemented with EGF growth factor was chosen, as we have shown this composition enriches for the differentiated cells that would be present in the small intestinal villus and the top of a colonic crypt over cells that are present in the base of a crypt, such as intestinal stem cells. Specifically, growth in media + EGF results in an increase in the goblet cell marker Mucin2 and the enterocyte marker sucrose isomaltose in organoids compared with organoids grown in WRN conditioned media, which is rich in growth factors because it contains both FBS as well as conditioned media-derived Wnt3a, R-spondin, and Noggin a. This is accompanied by a loss of expression of the stem cell marker leucine-rich repeat-containing G-protein coupled receptor 5 and the proliferation marker Ki67 in organoids grown in media + EGF compared with those grown in WRN conditioned media (Supplemental Fig.).

The question may arise about the relevance of an enclosed test system in which the organoid interior space corresponds to the lumen of the intestine. For example, the interior may accumulate shed cellular debris, which would otherwise be eliminated by lumenal content flow in normal gut physiology. Indeed, in vivo, the estimation is that it takes 4 to 5 days for a stem cell to differentiate to an enterocyte and move up the cryptvillus axis before being shed into the intestinal lumen (Clevers and Bevins, 2013). In organoid culture, accumulation of cellular debris in the organoid lumen is observed after long-term culture of 7-10 days (Sato et al., 2009). A consideration for the timing of our assay setup is the health of the organoids, which includes a lack of cellular debris in the lumen. As our assay runs for only 5 days, we do not see significant accumulation of shed enterocytes within the lumen of the organoids and therefore do not anticipate significant impact on the estimation of induction.

Cytochrome P450, UGT, and Transporter Inducibility in Ileal and Colon Organoids by Rifampin. To determine the utility of this model for understanding the expression of drug-metabolizing enzymes in the gut, a time course experiment with $100 \mu \mathrm{M}$ rifampin as the inducer was carried out for $0,4,24,48$, and 72 hours in ileal organoids (Fig. 1). These data showed robust induction response generally greater than 4fold from 24 to 72 hours. Based on these data, subsequent experiments for all inducers were carried out with a single 48-hour exposure time. The effect of increasing concentrations of rifampin on CYP3A4 induction in ileal and colon organoids is shown in Fig. 2, with each circle representing the response from organoids pooled from two Matrigel plugs. For both ileal and colon organoids, induction by rifampin was marked not only by statistically significant concentration dependence but also by marked variability (Fig. 2, A and C) across multiple experiments. The estimated $\mathrm{EC}_{50}$ and $\mathrm{E}_{\max }$ values for CYP3A4 were $3.75 \mu \mathrm{M}$ and 8.96 , respectively, with a Hill slope of 0.44 for ileal organoids. For colon organoids, estimated $\mathrm{EC}_{50}$ and $\mathrm{E}_{\max }$ values were $1.40 \mu \mathrm{M}$ and 11.3, respectively, with a Hill slope of 0.97 . Gray and blue circles represent data from two different donors, and these data indicated no marked donor-dependent differences in response. Next, the effect of rifampin on other selected transcripts (CYP1A1, CYP2B6, UGT1A1, Pgp, BCRP) is shown in comparison with CYP3A4 generated within the same experiment (except CYP1A1, which was an independent experiment) in ileal and colon organoids (Fig. 2, B and D). Unlike with CYP3A4, rifampin caused no increase in other transcripts.

Effect of Various Inducers on CYP3A4, CYP1A1, and CYP2B6 Induction in Ileal and Colon Organoids. To further understand induction responses in the organoid model, the effect of other prototypical inducers, such as CAR activators phenytoin and CITCO and the AhR activator omeprazole, on induction of CYP3A4 mRNA was 


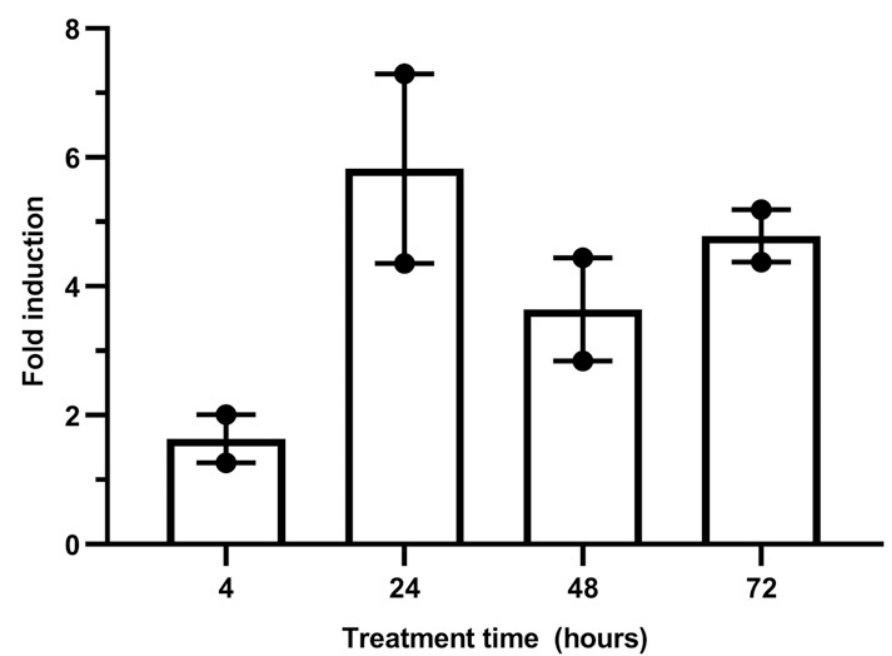

Fig. 1. Effect of incubation time and a single treatment of $100 \mu \mathrm{M}$ rifampin on the induction of CYP3A4 mRNA in ileal organoids from a single donor (donor 1). Data are from a single experiment and represent the means \pm range of two replicates, each consisting of an analysis of two pooled organoid-containing Matrigel plugs.

performed. As shown in Fig. 3 (Fig. 3A, ileal organoids; Fig. 3B, colon organoids), responses between tissues were remarkably similar despite relatively high variability when an induction response was indicated.
The effect of rifampin, phenytoin, and omeprazole on CYP1A1 mRNA induction is shown in Fig. 4. Although omeprazole treatment resulted in a concentration-dependent and variable increase in CYP1A1 mRNA in both ileal (geomean of 7.7-fold, 95\% CI 1.05-8.44, at $100 \mu \mathrm{M}$ omeprazole) and colon organoids (15.2-fold, 95\% CI 3.04-76.3, at $100 \mu \mathrm{M}$ omeprazole), other test compounds showed no evidence of induction. Figure 5 shows the effect of rifampin, phenytoin, CITCO, and omeprazole on CYP2B6 mRNA. In general, these compounds caused no induction of CYP2B6, with the exception of omeprazole, which appeared to induce CYP2B6 in a concentration-dependent manner in both ileal and colon organoids. One data point among the replicates for both rifampin $(100 \mu \mathrm{M})$ and CITCO indicated CYP2B6 induction, but this appeared to be spurious. Several attempts were made with various inducers to induce CYP1A2 in ileal and colon organoids, but this transcript was consistently found to be undetectable (e.g., Ct values $>35$ ).

CYP3A4 Enzyme Activity can be Detected in Intestinal Organoids. To determine whether the observed mRNA increases corresponded to functional protein activity, we measured the CYP3A4 enzyme activity in rifampin-treated ileal organoids. Figure 6A shows the accumulation of dehydronifedipine in incubations of rifampin-treated ileal organoids with 5 or $50 \mu \mathrm{M}$ nifedipine, a CYP3A4 probe substrate, after either 1- or 6-hour incubations in comparison with organoids treated with DMSO solvent vehicle only. Although enzyme activity was not detected after 1hour incubations, 6-hour incubations demonstrated CYP3A4-catalyzed dehydronifedipine metabolite formation. Using 5 and $50 \mu \mathrm{M}$ nifedipine
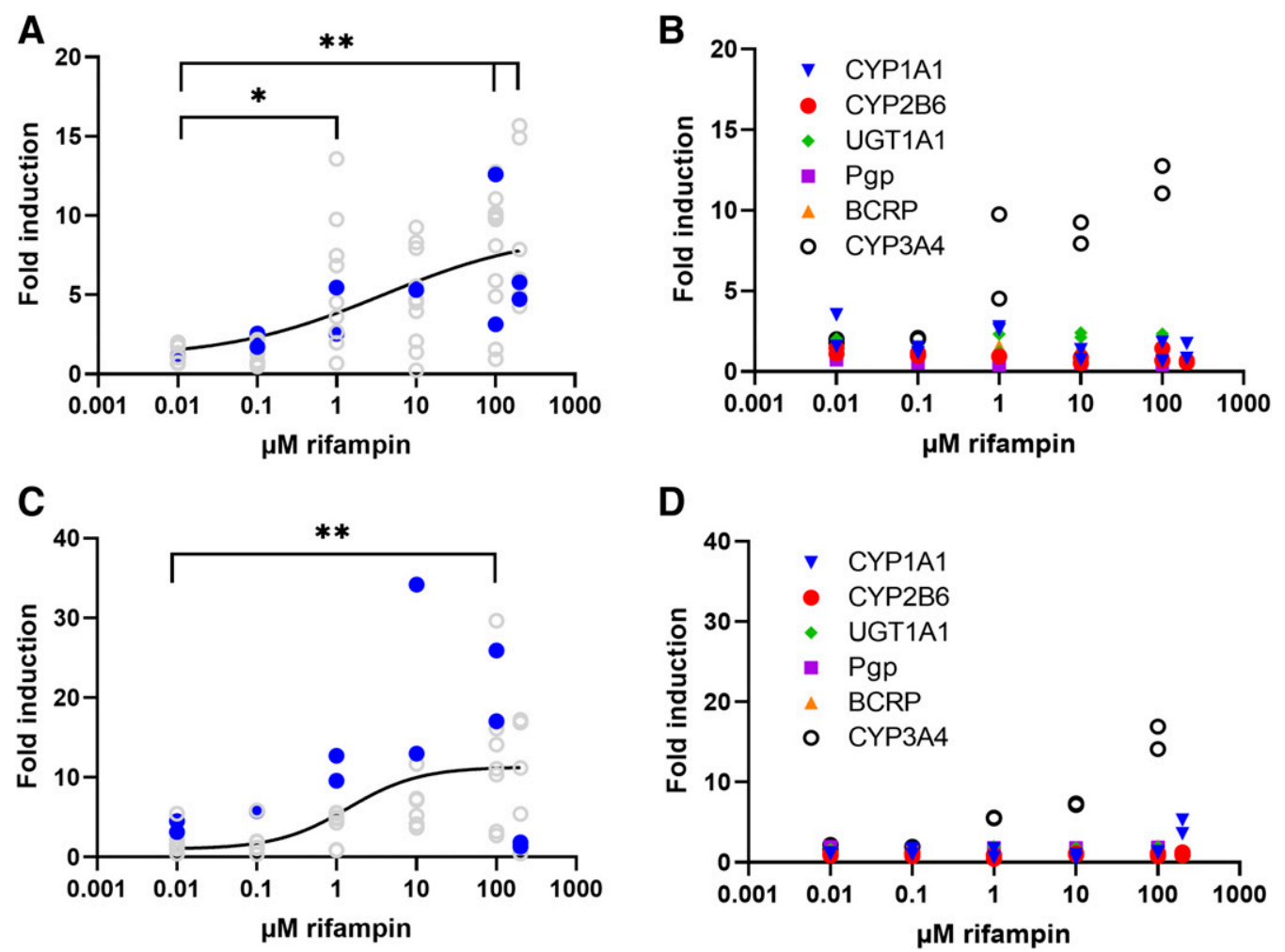

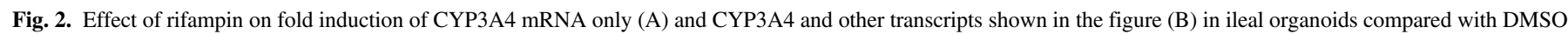

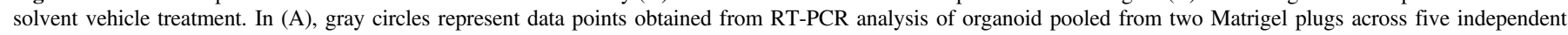

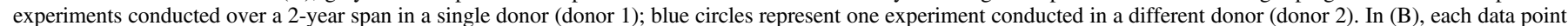

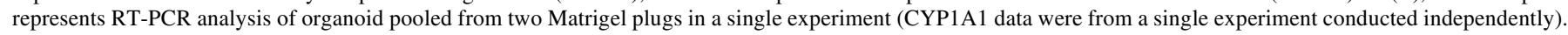

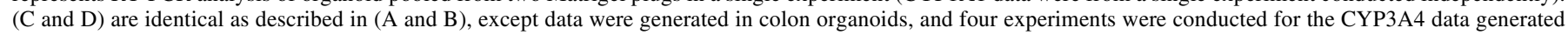

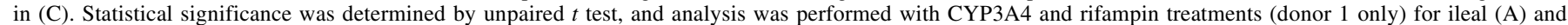

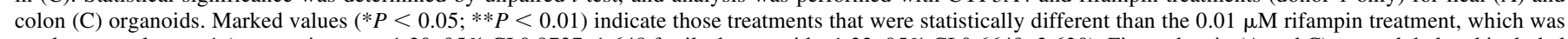

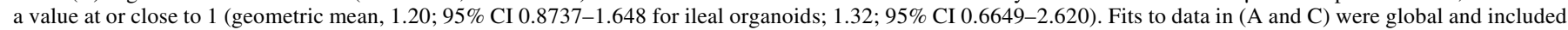
data points from both donors. 
A

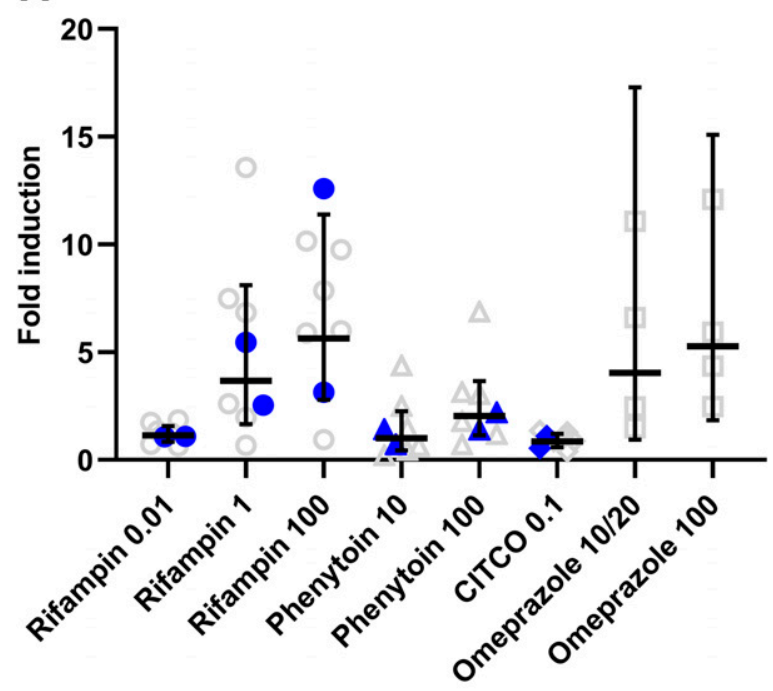

B

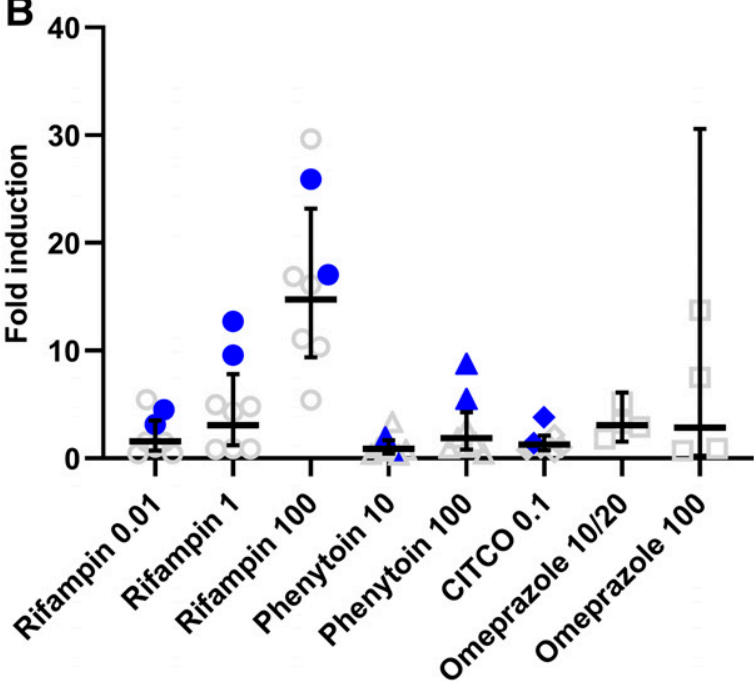

Fig. 3. Effect of various inducers on CYP3A4 mRNA induction in ileal (A) or colon (B) organoids. Concentration units are micromolar. Gray symbols represent data points obtained across two to four experiments in a single donor (donor 1); blue symbols represent one experiment conducted in a different donor (donor 2). Horizontal bars represent the geometric means, and vertical bars represent the 95\% confidence interval. Circles, triangles, diamonds, and squares correspond to treatment with rifampin, phenytoin, CITCO, and omeprazole, respectively.

as substrate rifampin treatment induced dehydronifedipine metabolite formation by 3.4- and 14.3-fold, respectively. It should be noted that formation of dehydronifedipine may not have been linear with incubation time, which may explain the fold induction disparity between the 5 and $50 \mu \mathrm{M}$ nifedipine incubations. Nevertheless, these data demonstrate that induction of CYP3A4 transcript (Fig. 6B) was accompanied by induction of CYP3A4 enzyme activity.

Cytotoxicity of Test Compounds. As the lack of transcriptional induction observed after treatment with some inducers might be explained by cytotoxicity, the effect of various treatments on organoid cell viability was measured in each study and is shown in Fig. 7. In all cases, organoids remained viable across treatments, with the exception of rifampin at $300 \mu \mathrm{M}$ and omeprazole at $200 \mu \mathrm{M}$. Therefore, cytochrome $\mathrm{P} 450$ induction data generated at these high concentrations were excluded from analysis.

PXR and CAR Expression in Ileal and Colon Organoids. To understand the mechanisms that may underlie the observed induction patterns in our organoid model, we investigated expression of PXR (NR1I2) and CAR (NR1I3) RNA in ileal and colon organoids from donor 1 at various times during the assay and after rifampin treatment (Fig. 8). The green squares represent the day 3 expression at the beginning of the assay when the inducers would be added, the blue triangle represents the expression in control samples on day 5 at the end of the assay, and the purple triangles represent the expression on day 5 after rifampin treatment. In all treatments, based on $\mathrm{Ct}$ values, expression of PXR is much higher than CAR in both ileal and colon organoids. PXR had $\mathrm{Ct}$ values ranging between 28 and 34, whereas CAR Ct values were all over 35 and in some cases undetectable, indicating lower levels or absence of expression in the model.
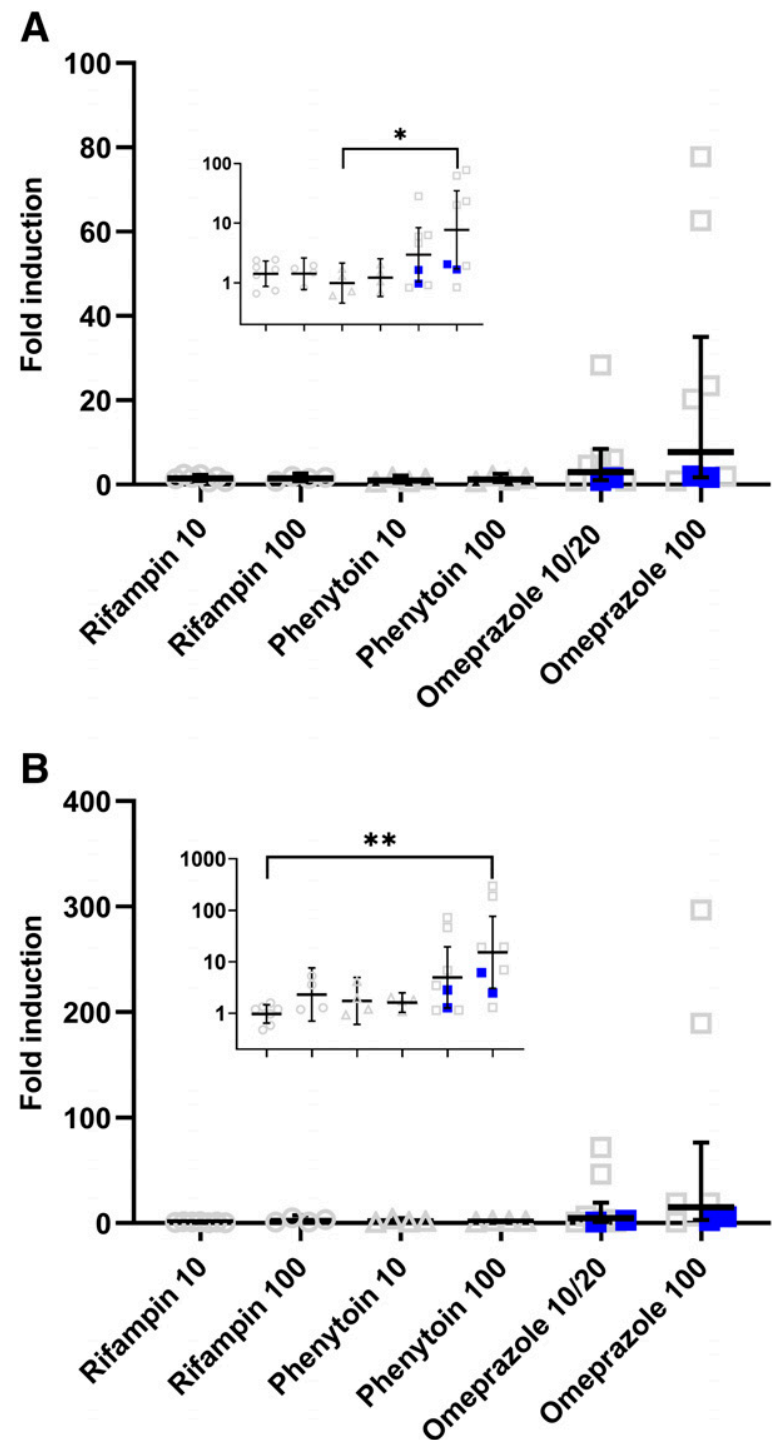

Fig. 4. Effect of various inducers on CYP1A1 mRNA fold induction over DMSO solvent vehicle in ileal (A) or colon (B) organoids. Concentration units are micromolar. Gray symbols represent data points obtained across two to four experiments in a single donor (donor 1); blue symbols represent one experiment conducted in a different donor (donor 2). Horizontal bars represent the geometric means, and vertical bars represent the $95 \%$ confidence interval. Insets: same data presented on $\log$ base 10 scale. Marked comparisons $(* P<0.05 ; * * P<0.01)$ indicate those treatments within donor 1 that were statistically different than the $10 \mu \mathrm{M}$ phenytoin treatment (ileal) or $10 \mu \mathrm{M}$ rifampin treatment (colon), which are data sets with mean values closest to 1 within each tissue (geometric mean, 0.9944; 95\% CI $0.4593-2.153$ for ileal organoids; 0.97 ; 95\% CI $0.6416-1.467$ for colon organoids). Circles, triangles, and squares correspond to treatment with rifampin, phenytoin, and omeprazole, respectively. 
A

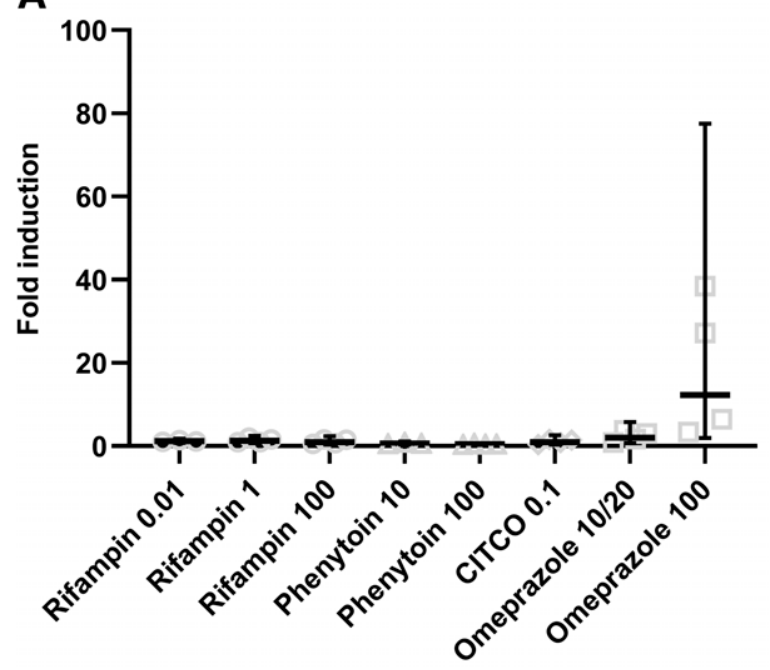

B

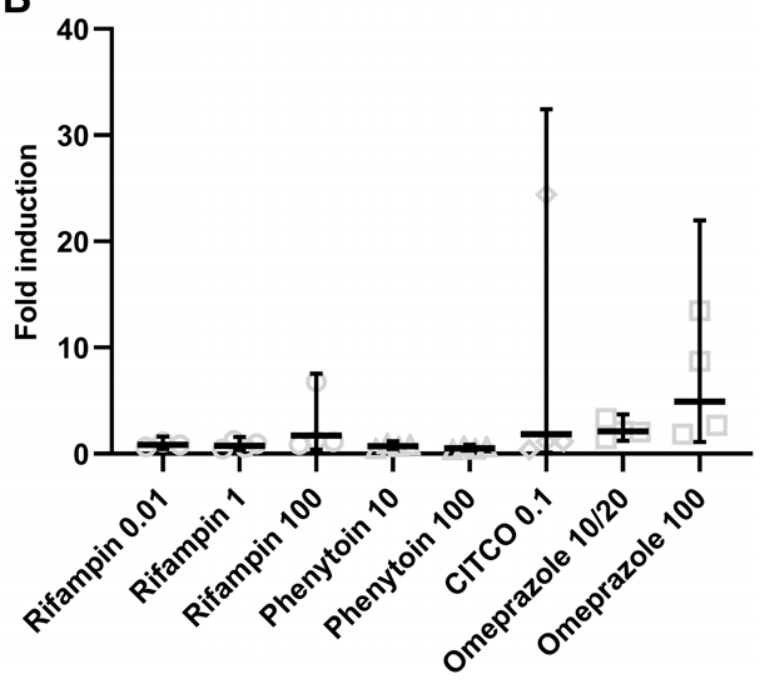

Fig. 5. Effect of various inducers on CYP2B6 mRNA induction in ileal (A) or colon (B) organoids. Concentration units are micromolar. Symbols represent data points obtained across two experiments in a single donor (donor 1). Horizontal bars represent the geometric means, and vertical bars represent the 95\% confidence interval. Using $0.01 \mu \mathrm{M}$ as a control value (geomean 1.198; 95\% CI 0.7939-1.807, ileal; 0.8546 , 95\% CI $0.4526-1.614$, colon), no treatment reached statistical significance (lowest adjusted $P$ value was 0.1867 with $100 \mu \mathrm{M}$ omeprazole, colon). Circles, triangles, diamonds, and squares correspond to treatment with rifampin, phenytoin, CITCO, and omeprazole, respectively.

\section{Discussion}

Organoids are multicellular microstructures exhibiting several desirable characteristics of an in vitro gut induction model. First, organoids (in this study) are grown from human tissue biopsies, generating a multi-cell type primary assay, with an expected improvement over immortalized cell culture systems (Sato et al., 2009; VanDussen et al., 2015). Second, organoid lines can be frozen and banked, enabling repeat testing of compounds from the same donor. Third, organoid cultures can be established in multiwell formats, facilitating concentration-response studies necessary to establish potency. Fourth, as demonstrated in this study, organoids can exhibit robust induction of mRNA and/or catalytic activity in response to compound treatment.

In contrast to iPS cell-derived organoids used by Onozato et al. (2018), we used organoids derived from biopsy specimens. These two culture methods differ in that biopsy-derived organoid lines require less time to grow and differentiate into organoids (3-5 days vs. up to 34 days). In addition, our stem cell-derived organoids comprised epithelial cells only versus iPS cell-derived organoids, which contain mesenchymal cell markers (i.e., $\alpha$-smooth muscle actin) (VanDussen et al., 2015; Onozato et al., 2018). Whereas Onozato et al. (2018) achieved $\sim 2$-fold CYP3A4 mRNA induction in response to rifampin treatment, we observed 9- to 11-fold induction in our model. Whether use of tissue stem cell-derived organoids or another aspect of our protocol enabled the higher induction response warrants further study.

In evaluating our model, we tested AhR, CAR, or PXR activators with a focus on rifampin as a PXR activator and well characterized CYP3A4 inducer. Drug concentrations were selected to mimic those that well induce target cytochrome P450 enzymes in hepatocyte models and were generally lower ( $\sim 2$ - to 10 -fold) than intestinal lumen concentrations estimated as dose per 250 milliliters (Zhang et al., 2008). In some cases, initially desired concentrations were adjusted lower to ensure adequate solubility (phenytoin) or avoid apparent cytotoxicity (omeprazole, rifampin). The concentration of CITCO was chosen to be selective for CAR while avoiding significant PXR activation (Maglich et al., 2003).
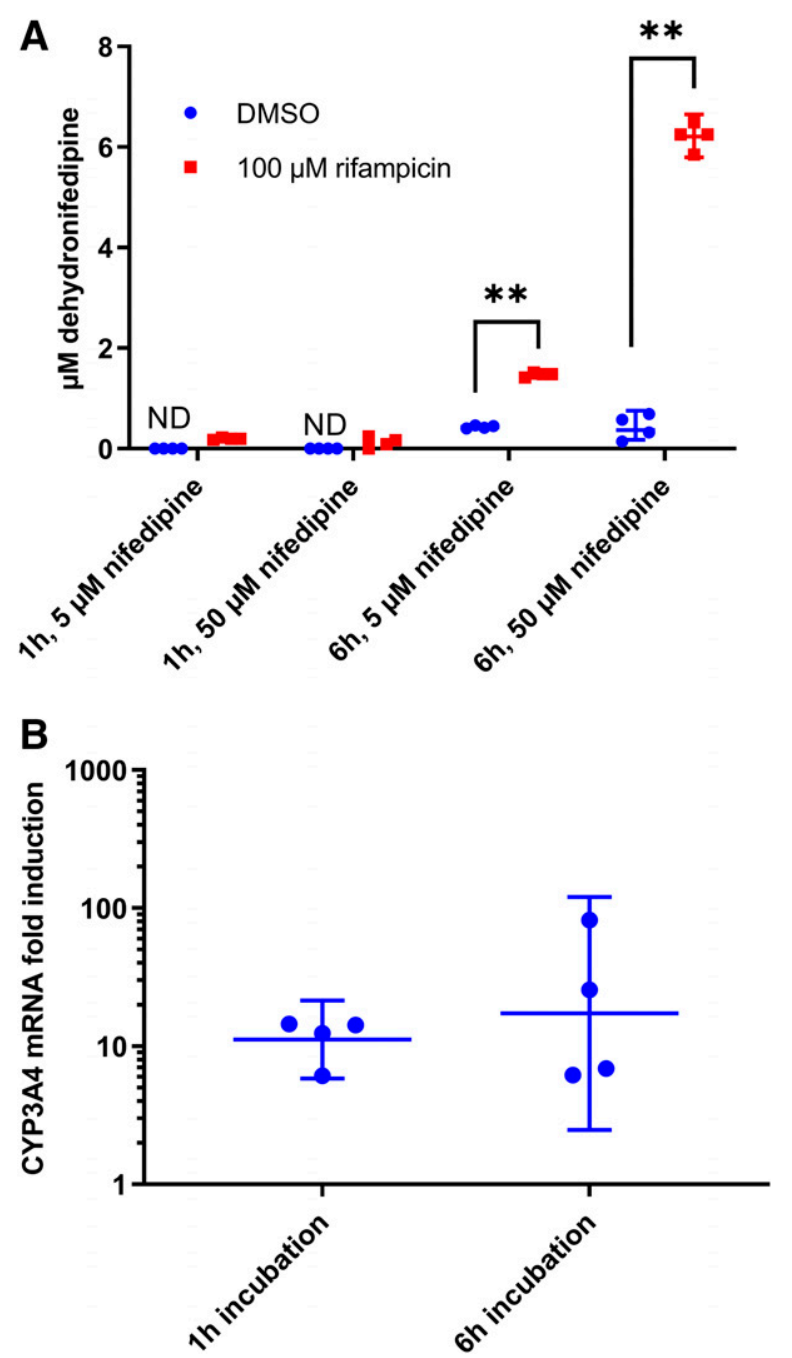

Fig. 6. Nifedipine oxidase activity in ileal organoids (donor 1) incubated with 5 or $50 \mu \mathrm{M}$ nifedipine for 1 or 6 hours after a 48-hour treatment with $100 \mu \mathrm{M}$ rifampin or DMSO solvent vehicle only (A). ND, not detected. Marked comparisons $(* * P<$ 0.01 ) indicate statistical difference. (B) shows CYP3A4 fold induction observed in the same organoids used for nifedipine oxidase activity experiments after incubation with nifedipine for the time indicated. In both panels, horizontal bars represent the geometric means of four replicates (each replicate consistent of a pool of two organoid plugs), and vertical bars represent the $95 \%$ confidence interval. 
A

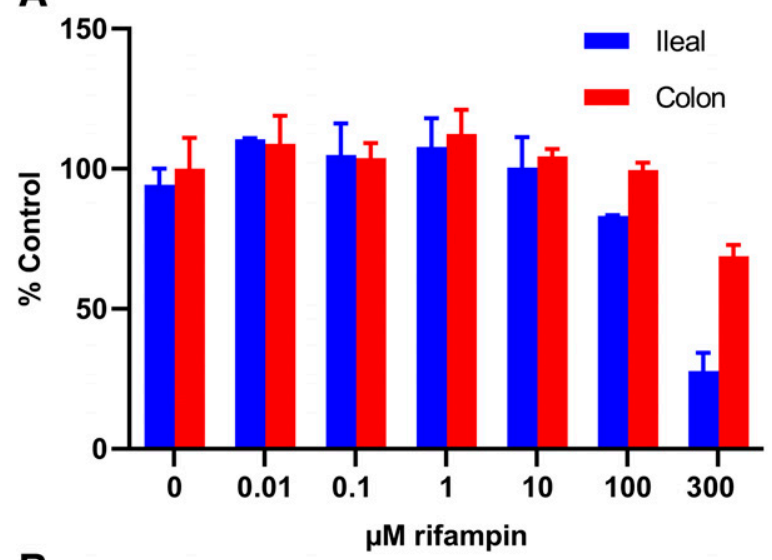

B

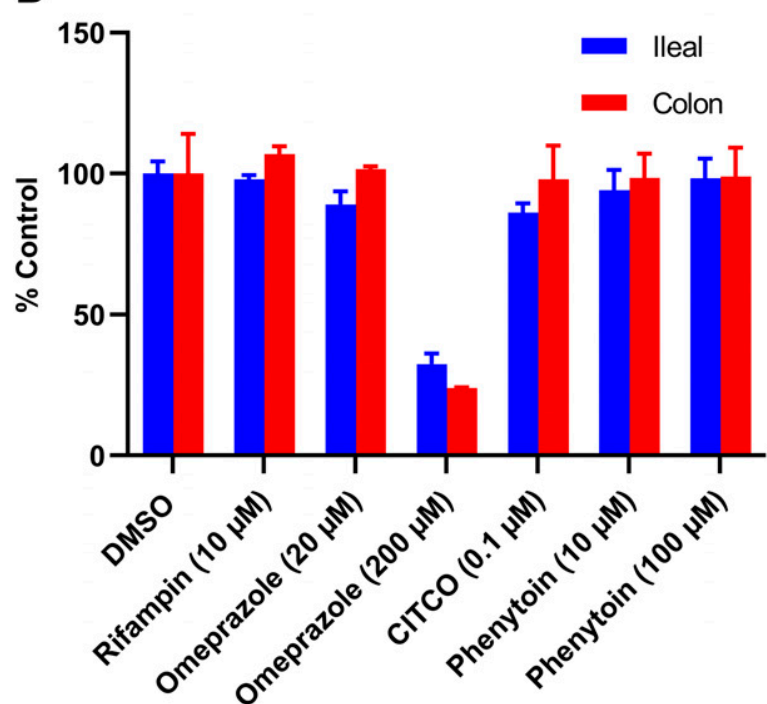

Fig. 7. Effect of rifampin (A) or various test compounds (B) on organoid cell viability (CellTiter GLO 3D). Bars represent the mean of two replicates; error bars indicate the range of the two values.

With rifampin, $\mathrm{EC}_{50}$ values in ileal and colon organoids were 3.75 and $1.4 \mu \mathrm{M}$, respectively. These compare with typical findings in hepatocytes, showing rifampin $\mathrm{EC}_{50}$ values generally ranging between 0.1 and $1 \mu \mathrm{M}$ (https://didb.druginteractionsolutions.org/, accessed November 17, 2020). Complicating the estimation of kinetic parameters was the relatively high inter- and intraexperimental variability observed. Data variability did not seem to be donor-dependent, as testing in a second donor gave similar results (indicated as blue symbols in plots containing gray symbols) and may be the result of the more complex and cellularly diverse organoid system. Another consideration in interpreting differences in kinetic parameters is that intracellular exposure may vary compared with hepatocytes, perhaps because of the organoid architecture (inability to directly access the apical side interior) or relative expression of uptake and efflux transporters.

We also evaluated the effect of rifampin on other transcripts. Data in Fig. 2 show that although CYP3A4 was induced in a concentrationdependent manner, CYP1A1, CYP2B6, UGT1A1, Pgp, or BCRP exhibited no induction. Absent CYP1A1 induction might be expected, since rifampin is not known to induce this enzyme. Lack of UGT1A1 induction was also unsurprising, as rifampin did not increase UGT1A1catalyzed estradiol 3-glucuronidation in hepatocytes (Soars et al., 2004). However, one might expect induction of CYP2B6, since rifampin readily induces CYP2B6 in hepatocytes (discussed further below).
Induction of Pgp mRNA was not observed, which contrasts with findings that mRNA was induced in duodenal biopsies in individuals given rifampin (Greiner et al., 1999; Larsen et al., 2007; Brueck et al., 2019). In the aforementioned biopsy studies, the fold induction was rather modest ( $\sim 1.3$ - to 3.5-fold). Absence of Pgp induction is possibly attributable to differences in the relative percentage of enterocytes (i.e., the cell type believed responsive to inducing agents) in organoids compared with biopsy tissue. Absence of rifampin induction of BCRP in organoids agrees with previous reports in intestinal biopsies (Brueck et al., 2019). It should be noted that the percentage of biopsy mass composed of enterocytes was not reported in those studies, suggesting that the fold induction by rifampin may be underestimated.
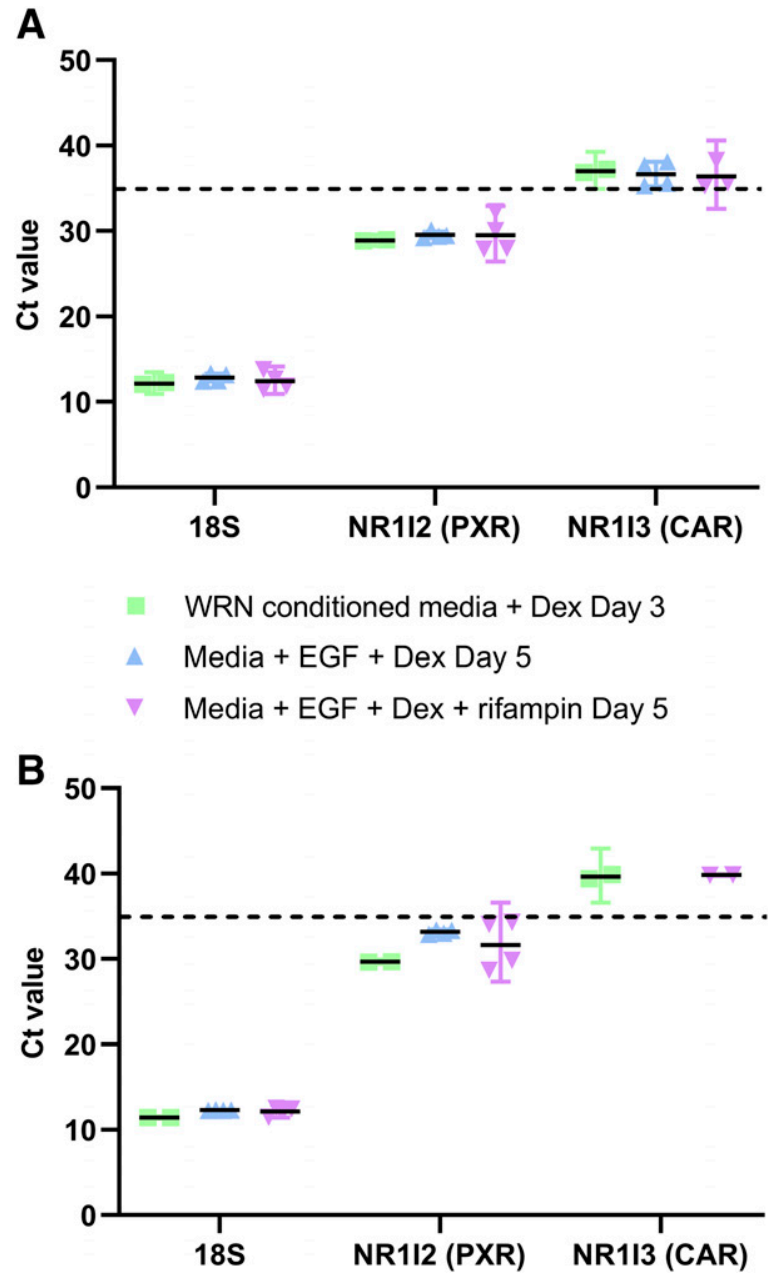

Fig. 8. PXR expression is higher in ileal and colon organoids compared with CAR expression. To determine whether PXR and CAR were expressed in ileal and colon organoids during the conditions used for assays, RNA was extracted and expression of 18S (housekeeping gene), NR1I2 (PXR), and NR1I3 (CAR) levels quantified by RT-PCR, Specifically RNA was extracted from ileal and colon organoids from donor 1 after 3 days of growth in WRN conditioned media (green squares represent the day 3 expression at the beginning of the assay when the inducers would be added) after the organoids were subsequently grown in media + EGF for 2 days (blue triangles represent the expression in control samples on day 5 at the end of the assay) and after a subsequent growth in media + EGF + rifampin (purple triangles represent the expression on day 5 after rifampin treatment at the end of the assay). In all treatments, based on $\mathrm{Ct}$ values, expression of PXR is significantly higher than CAR in both ileal (A) and colon (B) organoids. PXR Ct values ranged from 28 to 34, whereas CAR Ct values were all over 35 and were in some cases undetectable, indicating lower levels or absence of expression in the model. Each data point represents the data from two pooled wells of organoids at the indicated time point, and data from two independent studies performed in duplicate (four data points total) are shown on the graph. Dex, dexamethasone. 
The effect of compounds that predominantly activate CAR (phenytoin or CITCO) or AhR (omeprazole) on CYP3A4 expression was examined (Fig. 2). Phenytoin appeared to show relatively weak, concentrationdependent induction, whereas $0.1 \mu \mathrm{M}$ CITCO gave no effect. Since both compounds readily induce CYP3A4 in hepatocytes, which highly express CAR, the findings suggested the possibility that CAR mechanisms are absent in organoids. By RT-PCR, we found CAR levels were about 20-fold lower than PXR in untreated organoids. Subsequent inspection of an RNA-sequencing data base in tissues from $>180$ cadavers revealed CAR expression is very low in transverse colon and terminal ileum tissue compared with PXR expression, whereas CAR expression is comparable to PXR in liver (Fig. 9). These data are also in agreement with Brueck et al. (2019), showing much lower CAR expression in duodenal biopsies. This may in part explain findings that efavirenz and carbamazepine, two CAR preferential activators (Faucette et al., 2007), exhibit induction in liver but not intestine (Mouly et al., 2002; Giessmann et al., 2004; Meyer zu Schwabedissen et al., 2012). Together, these data suggest compounds inducing CYP3A4 by a CARmediated mechanism may not induce CYP3A4 in gut. This has implications for drug development. For example, some compounds could be spared from autoinduction of CYP3A4 clearance in gut or be less prone to induce clearance of comedications that undergo significant CYP3A4-mediated gut metabolism. Omeprazole induced CYP3A4 (up to 5.3-fold, geomean, $n=4$ experiments), which is in line with previous reports of omeprazole as a PXR activator and CYP3A4 inducer (Novotna and Dvorak, 2014) in human hepatocytes.

We also examined CYP1A1 and CYP1A2 induction response by activators and nonactivators of AhR. In both ileal and colon organoids, we found CYP1A1 mRNA was highly induced by omeprazole. Consistent with this observation, CYP1A1 protein is detected in meaningful quantities in human small intestinal microsomes and is known to be inducible in this tissue (Paine et al., 2006; Fontana et al., 1999; Zhang et al., 1999). As anticipated, rifampin and phenytoin failed to induce CYP1A1 in either ileal or colon organoids. Omeprazole did not induce CYP1A2 mRNA in organoids, nor was the transcript detected, agreeing with reports showing CYP1A2 transcripts or protein undetectable in gut (Paine et al., 2006; Thelen and Dressman, 2009). We did not detect induction of CYP2B6 by rifampin, phenytoin, and CITCO,

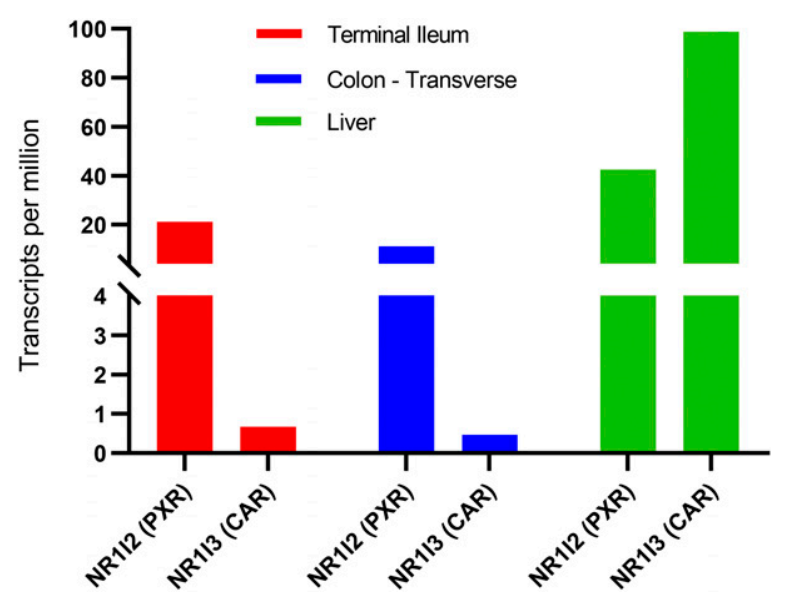

Fig. 9. RNA-sequencing analysis of PXR and CAR gene expression from tissues in $>180$ cadavers (data obtained from the Genotype-Tissue Expression project data base, https://gtexportal.org/home/) showing >20-fold less CAR expression in terminal ileum and colon compared with PXR. On the other hand, CAR expression was $>2.3$-fold higher than PXR in liver tissue. Values represent the median from analysis of 187 (ileum), 226 (liver), and 406 (colon) donors. It should be noted that transcripts per million values are not comparable between tissues, since the value depends on the total number of transcripts recovered from tissues. respectively, in line with reports that CYP2B6 is not expressed in gut mucosa (Zhang et al., 1999; Paine et al., 2006). Interestingly, the CYP2B6 transcript was readily detectable, with $\mathrm{Ct}$ values ranging from $\sim 25$ to $\sim 29$ in ileal and colon organoids across the various treatments. This suggests that absence of CYP2B6 protein expression in mucosa may be related to unavailability of cellular systems permitting CYP2B6 translation.

Although we evaluated our model with activators of AhR, CAR, and PXR, it should be noted that lesser known or atypical pathways that regulate $\mathrm{P} 450$ and transporter expression may be present in this tissue and may exhibit differing relative importance compared with liver (Dixit et al., 2005; Zheng et al., 2012; Gerbal-Chaloin et al., 2014; Müller et al., 2017). We did not examine inducibility originating from the various cell types within the organoids. However, as enterocytes are present in the ileal organoids, and enterocytes exhibit relatively high CYP3A4 expression (McKinnon et al., 1995; Zhang et al., 1999; Glaeser et al., 2005), it can be expected that the induction response we measured originated primarily from enterocytes. Similarly, one might expect the same for the colonocyte-like cells in colon organoids. Within the small intestine, the upper portion (jejunum, duodenum) typically exhibits the highest concentration of P450 (Zhang et al., 1999), whereas the distal portion (ileum) exhibits higher Pgp expression (Mouly and Paine, 2003). Colon epithelium is not considered a significant contributor to drug metabolism; absorbing surface area is very low-about $0.2 \%$ that of the combined surface area of ileum and duodenum (Kararli, 1995)_and P450 and other drug-metabolizing activities are generally low or undetectable (Peters et al., 1991; van de Kerkhof et al., 2008). Therefore, although the response to inducers in colon organoids in our study were strikingly similar to ileal organoids, both qualitatively and quantitatively (e.g., Figs. 2-5), studies in jejunal and duodenal organoids might be needed to fully predict induction responses in the gastrointestinal tract.

In sum, we have characterized selected cytochrome P450 and transporter induction properties of a novel and physiologically relevant gut model. Data demonstrate that organoids derived from human adult ileal and colonic stem cells are a potentially promising test system to evaluate cytochrome $\mathrm{P} 450$ induction. Organoids appear to recapitulate expected intestinal induction responses, such as induction of CYP3A4 and CYP1A1, but not CYP1A2 or CYP2B6. Another notable finding is the absence of efflux transporter mRNA induction. CAR mRNA is expressed at levels much lower than PXR in organoids and intestinal tissue, in contrast to liver. Coupled with absence of CYP3A4 induction by CAR activators in this model, our data suggest that genes regulated by CAR appear to be noninducible and may explain, at least in part, compartmental CYP3A4 induction by some compounds that are predominantly CAR activators. This indicates that the model could be used to de-risk drug candidates identified as CAR-mediated inducers. Limitations to this organoid model include the relative lack of commercial accessibility and relatively high intraexperimental data variability. Future efforts should be aimed at improving assay variability and develop methods to scale data to in vivo response, possibly enabling application to physiologically-based pharmacokinetic modeling.

\section{Acknowledgments}

The authors would like to thank Sudiksha Chaulagain for experimental support, Hardikkumar Patel for nifedipine oxidase assay bioanalytical support, Kelly Desino for management support, Edda Fiebiger for scientific discussion, and Julius Enoru for critical review and comments on the manuscript. All contributors are employees of AbbVie.

\section{Authorship Contributions}

Participated in research design: Stresser, Sun, Wilson.

Conducted experiments: Sun, Wilson.

Performed data analysis: Stresser, Sun, Wilson.

Wrote or contributed to the writing of the manuscript: Stresser, Sun, Wilson. 


\section{References}

Alqahtani S, Bukhari I, Albassam A, and Alenazi M (2018) An update on the potential role of intestinal first-pass metabolism for the prediction of drug-drug interactions: the role of PBPK modeling. Expert Opin Drug Metab Toxicol 14:625-634.

Brück S, Strohmeier J, Busch D, Drozdzik M, and Oswald S (2017) Caco-2 cells - expression, regulation and function of drug transporters compared with human jejunal tissue. Biopharm Drug Dispos 38:115-126.

Brueck S, Bruckmueller H, Wegner D, Busch D, Martin P, Oswald S, Cascorbi I, and Siegmund W (2019) Transcriptional and post-transcriptional regulation of duodenal P-glycoprotein and MRP2 in healthy human subjects after chronic treatment with rifampin and carbamazepine. Mol Pharm 16:3823-3830.

Clevers HC and Bevins CL (2013) Paneth cells: maestros of the small intestinal crypts. Annu Rev Physiol 75:289-311

Dixit SG, Tirona RG, and Kim RB (2005) Beyond CAR and PXR. Curr Drug Metab 6:385-397.

Drozdzik M, Busch D, Lapczuk J, Müller J, Ostrowski M, Kurzawski M, and Oswald S (2018) Protein abundance of clinically relevant drug-metabolizing enzymes in the human liver and intestine: a comparative analysis in paired tissue specimens. Clin Pharmacol Ther 104:515-524.

Fatehullah A, Tan SH, and Barker N (2016) Organoids as an in vitro model of human development and disease. Nat Cell Biol 18:246-254.

Faucette SR, Zhang TC, Moore R, Sueyoshi T, Omiecinski CJ, LeCluyse EL, Negishi M, and Wang H (2007) Relative activation of human pregnane $X$ receptor versus constitutive androstane receptor defines distinct classes of CYP2B6 and CYP3A4 inducers. J Pharmacol Exp Ther 320:72-80.

Fontana RJ, Lown KS, Paine MF, Fortlage L, Santella RM, Felton JS, Knize MG, Greenberg A, and Watkins PB (1999) Effects of a chargrilled meat diet on expression of CYP3A, CYP1A, and P-glycoprotein levels in healthy volunteers. Gastroenterology 117:89-98.

Fowler S, Chen WLK, Duignan DB, Gupta A, Hariparsad N, Kenny JR, Lai WG, Liras J, Phillips JA, and Gan J (2020) Microphysiological systems for ADME-related applications: current status and recommendations for system development and characterization. Lab Chip 20:446-467.

Gerbal-Chaloin S, Dumé A-S, Briolotti P, Klieber S, Raulet E, Duret C, Fabre J-M, Ramos J, Maurel P, and Daujat-Chavanieu M (2014) The WNT/B-catenin pathway is a transcriptional regulator of CYP2E1, CYP1A2, and aryl hydrocarbon receptor gene expression in primary human hepatocytes. Mol Pharmacol 86:624-634.

Galetin A, Gertz M, and Houston JB (2010) Contribution of intestinal cytochrome p450-mediated metabolism to drug-drug inhibition and induction interactions. Drug Metab Pharmacokinet 25: $28-47$.

Giessmann T, May K, Modess C, Wegner D, Hecker U, Zschiesche M, Dazert P, Grube M, Schroeder E, Warzok R, et al. (2004) Carbamazepine regulates intestinal P-glycoprotein and multidrug resistance protein MRP2 and influences disposition of talinolol in humans. Clin Pharmacol Ther 76:192-200.

Glaeser H, Drescher S, Eichelbaum M, and Fromm MF (2005) Influence of rifampicin on the expression and function of human intestinal cytochrome P450 enzymes. Br J Clin Pharmacol 59: 199-206.

Greiner B, Eichelbaum M, Fritz P, Kreichgauer HP, von Richter O, Zundler J, and Kroemer HK (1999) The role of intestinal P-glycoprotein in the interaction of digoxin and rifampin. J Clin Invest 104:147-153.

GTEx Consortium (2013) The genotype-tissue expression (GTEx) project. Nat Genet 45:580-585.

Gupta A, Mugundu GM, Desai PB, Thummel KE, and Unadkat JD (2008) Intestinal human colon adenocarcinoma cell line LS180 is an excellent model to study pregnane X receptor, but not constitutive androstane receptor, mediated CYP3A4 and multidrug resistance transporter 1 induction: studies with anti-human immunodeficiency virus protease inhibitors. Drug Metab Dispos 36:1172-1180.

Hartley DP, Dai X, Yabut J, Chu X, Cheng O, Zhang T, He YD, Roberts C, Ulrich R, Evers R, et al. (2006) Identification of potential pharmacological and toxicological targets differentiating structural analogs by a combination of transcriptional profiling and promoter analysis in LS-180 and Caco-2 adenocarcinoma cell lines. Pharmacogenet Genomics 16:579-599.

Kararli TT (1995) Comparison of the gastrointestinal anatomy, physiology, and biochemistry of humans and commonly used laboratory animals. Biopharm Drug Dispos 16:351-380.

Kasendra M, Luc R, Yin J, Manatakis DV, Kulkarni G, Lucchesi C, Sliz J, Apostolou A, Sunuwar L, Obrigewitch J, et al. (2020) Duodenum Intestine-Chip for preclinical drug assessment in a human relevant model. eLife $\mathbf{9}:$ e50135.

Larsen UL, Hyldahl Olesen L, Guldborg Nyvold C, Eriksen J, Jakobsen P, Østergaard M, Autrup $\mathrm{H}$, and Andersen V (2007) Human intestinal P-glycoprotein activity estimated by the model substrate digoxin. Scand J Clin Lab Invest 67:123-134.

Li AP, Alam N, Amaral K, Ho MD, Loretz C, Mitchell W, and Yang Q (2018) Cryopreserved human intestinal mucosal epithelium: a novel in vitro experimental system for the evaluation of enteric drug metabolism, P450 induction, and enterotoxicity. Drug Metab Dispos 46:1562-1571.

Madden LR, Nguyen TV, Garcia-Mojica S, Shah V, Le AV, Peier A, Visconti R, Parker EM, Presnell SC, Nguyen DG, et al. (2018) Bioprinted 3D primary human intestinal tissues model aspects of native physiology and ADME/Tox functions. iScience 2:156-167.

Maglich JM, Parks DJ, Moore LB, Collins JL, Goodwin B, Billin AN, Stoltz CA, Kliewer SA, Lambert MH, Willson TM, et al. (2003) Identification of a novel human constitutive androstane receptor (CAR) agonist and its use in the identification of CAR target genes. J Biol Chem $\mathbf{2 7 8}$ $17277-17283$.

McKinnon RA, Burgess WM, Hall PM, Roberts-Thomson SJ, Gonzalez FJ, and McManus ME (1995) Characterisation of CYP3A gene subfamily expression in human gastrointestinal tissues. Gut 36:259-267.
Meyer zu Schwabedissen HE, Oswald S, Bresser C, Nassif A, Modess C, Desta Z, Ogburn ET, Marinova M, Lütjohann D, Spielhagen C, et al. (2012) Compartment-specific gene regulation of the CAR inducer efavirenz in vivo [published correction appears in Clin Pharmacol Ther (2013) 93:129]. Clin Pharmacol Ther 92:103-111.

Miyoshi H and Stappenbeck TS (2013) In vitro expansion and genetic modification of gastrointestinal stem cells in spheroid culture. Nat Protoc 8:2471-2482.

Mouly S, Lown KS, Kornhauser D, Joseph JL, Fiske WD, Benedek IH, and Watkins PB (2002) Hepatic but not intestinal CYP3A4 displays dose-dependent induction by efavirenz in humans. Clin Pharmacol Ther 72:1-9.

Mouly S and Paine MF (2003) P-glycoprotein increases from proximal to distal regions of human small intestine. Pharm Res 20:1595-1599.

Müller J, Keiser M, Drozdzik M, and Oswald S (2017) Expression, regulation and function of intestinal drug transporters: an update. Biol Chem 398:175-192.

Novotna A and Dvorak Z (2014) Omeprazole and lansoprazole enantiomers induce CYP3A4 in human hepatocytes and cell lines via glucocorticoid receptor and pregnane $\mathrm{X}$ receptor axis. PLoS One 9:e105580.

Oda S, Kato Y, Hatakeyama M, Iwamura A, Fukami T, Kume T, Yokoi T, and Nakajima M (2017) Evaluation of expression and glycosylation status of UGT1A10 in Supersomes and intestinal epithelial cells with a novel specific UGT1A10 monoclonal antibody. Drug Metab Dispos 45: 1027-1034.

Onozato D, Yamashita M, Nakanishi A, Akagawa T, Kida Y, Ogawa I, Hashita T, Iwao T, and Matsunaga T (2018) Generation of intestinal organoids suitable for pharmacokinetic studies from human induced pluripotent stem cells. Drug Metab Dispos 46:1572-1580.

Paine MF, Hart HL, Ludington SS, Haining RL, Rettie AE, and Zeldin DC (2006) The human intestinal cytochrome P450 "pie". Drug Metab Dispos 34:880-886.

Pascussi JM, Drocourt L, Fabre JM, Maurel P, and Vilarem MJ (2000) Dexamethasone induces pregnane $\mathrm{X}$ receptor and retinoid $\mathrm{X}$ receptor-alpha expression in human hepatocytes: synergistic increase of CYP3A4 induction by pregnane X receptor activators. Mol Pharmacol 58:361-372.

Peters WH, Kock L, Nagengast FM, and Kremers PG (1991) Biotransformation enzymes in human intestine: critical low levels in the colon? Gut 32:408-412.

Sato T, Stange DE, Ferrante M, Vries RG, Van Es JH, Van den Brink S, Van Houdt WJ, Pronk A, Van Gorp J, Siersema PD, et al. (2011) Long-term expansion of epithelial organoids from human colon, adenoma, adenocarcinoma, and Barrett's epithelium. Gastroenterology 141:1762-1772.

Sato T, Vries RG, Snippert HJ, van de Wetering M, Barker N, Stange DE, van Es JH, Abo A Kujala P, Peters PJ, et al. (2009) Single Lgr5 stem cells build crypt-villus structures in vitro without a mesenchymal niche. Nature 459:262-265.

Schmiedlin-Ren P, Thummel KE, Fisher JM, Paine MF, and Watkins PB (2001) Induction of CYP3A4 by 1 alpha,25-dihydroxyvitamin D3 is human cell line-specific and is unlikely to involve pregnane X receptor. Drug Metab Dispos 29:1446-1453.

Schuetz EG, Beck WT, and Schuetz JD (1996) Modulators and substrates of P-glycoprotein and cytochrome P4503A coordinately up-regulate these proteins in human colon carcinoma cells. Mol Pharmacol 49:311-318.

Soars MG, Petullo DM, Eckstein JA, Kasper SC, and Wrighton SA (2004) An assessment of udpglucuronosyltransferase induction using primary human hepatocytes. Drug Metab Dispos 32: 140-148.

Thelen K and Dressman JB (2009) Cytochrome P450-mediated metabolism in the human gut wall. J Pharm Pharmacol 61:541-558.

Thummel KE, Brimer C, Yasuda K, Thottassery J, Senn T, Lin Y, Ishizuka H, Kharasch E, Schuetz J, and Schuetz E (2001) Transcriptional control of intestinal cytochrome P-4503A by $1 \alpha, 25$ dihydroxy vitamin D3. Mol Pharmacol 60:1399-1406.

van de Kerkhof EG, de Graaf IAM, Ungell A-LB, and Groothuis GMM (2008) Induction of metabolism and transport in human intestine: validation of precision-cut slices as a tool to study induction of drug metabolism in human intestine in vitro. Drug Metab Dispos 36:604-613.

VanDussen KL, Marinshaw JM, Shaikh N, Miyoshi H, Moon C, Tarr PI, Ciorba MA, and Stappenbeck TS (2015) Development of an enhanced human gastrointestinal epithelial culture system to facilitate patient-based assays. Gut 64:911-920.

VanDussen KL, Sonnek NM, and Stappenbeck TS (2019) L-WRN conditioned medium for gastrointestinal epithelial stem cell culture shows replicable batch-to-batch activity levels across multiple research teams. Stem Cell Res (Amst) 37:101430.

Varma MV, Obach RS, Rotter C, Miller HR, Chang G, Steyn SJ, El-Kattan A, and Troutman MD (2010) Physicochemical space for optimum oral bioavailability: contribution of human intestinal absorption and first-pass elimination. J Med Chem 53:1098-1108.

Zhang L, Zhang YD, Strong JM, Reynolds KS, and Huang S-M (2008) A regulatory viewpoint on transporter-based drug interactions. Xenobiotica 38:709-724.

Zhang QY, Dunbar D, Ostrowska A, Zeisloft S, Yang J, and Kaminsky LS (1999) Characterization of human small intestinal cytochromes P-450. Drug Metab Dispos 27:804-809.

Zheng XE, Wang Z, Liao MZ, Lin YS, Shuhart MC, Schuetz EG, and Thummel KE (2012) Human PXR-mediated induction of intestinal CYP3A4 attenuates $1 \alpha, 25$-dihydroxyvitamin $\mathrm{D}_{3}$ function in human colon adenocarcinoma LS180 cells. Biochem Pharmacol 84:391-401.

Zhang Y and Benet LZ (2001) The gut as a barrier to drug absorption: combined role of cytochrome P450 3A and P-glycoprotein. Clin Pharmacokinet 40:159-168.

Address correspondence to: David M. Stresser, Drug Metabolism and Pharmacokinetics, AbbVie, 1 North Waukegan Rd., North Chicago, IL 60064. E-mail: David.Stresser@abbvie.com 